\title{
Immune impairment in HIV infection: Existence of risky and immunodeficiency thresholds
}

\author{
Shingo Iwami ${ }^{a *}$, Tomoyuki Miura $^{b}$, Shinji Nakaoka ${ }^{c, d}$, \\ Yasuhiro Takeuchi ${ }^{a}$ \\ Graduate School of Science and Technology, Shizuoka University, Japan ${ }^{a}$, \\ Laboratory of Primate Model, Institute for Virus Research, Kyoto University, Japan ${ }^{b}$, \\ Aihara Complexity Modelling Project, ERATO, JST, The University of Tokyo, Japan ${ }^{c}$, \\ Graduate School of Mathematical Sciences, The University of Tokyo, Japan ${ }^{d}$
}

\begin{abstract}
Results of several studies show that some DC populations are susceptible to HIV. Modulation of DCs by HIV infection, in particular interference of the antigen-presenting function of DCs, is a key aspect in viral pathogenesis and contributes to viral evasion from immunity because the loss of the DC function engenders some impairment effects for a proliferation of CTL responses, which play an important role in the immune response to HIV. As described herein, we use a simple mathematical model to examine virus-immune dynamics over the course of HIV infection in the context of the immune impairment effects. A decrease of the DC number and function during the course of HIV-1 infection is observed. Therefore, we simply assumed that the immune impairment rate increases over the HIV infection. Under the assumption, four processes of the disease progression dynamics of our model are classifiable according to their virological properties. It is particularly interesting a typical disease progression presents a "Risky threshold" and an "Immunodeficiency threshold". Regarding the former, the immune system might collapse when the impairment rate of HIV exceeds a threshold value (which corresponds to a transcritical bifurcation point). For the latter, the immune system always collapses when the impairment rate exceeds the value (which corresponds to a saddle-node bifurcation point). To test our theoretical framework, we investigate the existence and distribution of these thresholds in 10 patients.
\end{abstract}

Keywords: HIV infection; Dendritic cell; Mathematical model; Immune impairment; Immunodeficiency; Bistability; Saddle-node bifurcation; Transcritical bifurcation

\footnotetext{
${ }^{*}$ Corresponding author. E-mail: dsiwami@ipc.shizuoka.ac.jp
} 


\section{Introduction}

A basic principle of immunology has largely been ignored in defining immune parameters of HIV infection: the role of professional antigen presenting cells (APCs) dendritic cells (DCs), monocytes/macrophages, and B lymphocytes [34]. However, these cells play an important role in immune responses (see Fig. 1). In fact, DCs are positioned geographically as sentinels, detecting "danger signals" and linking innate and adaptive immune responses [10,34]. These cells mature and migrate to the secondary lymphoid tissue after they encounter HIV in the periphery [10, 19]. The DC maturation process involves increasing antigen presentation on major histocompatibility complex (MHC) molecules, and upregulating co-stimulatory molecules $[15,19]$. The mature DCs present HIV peptides to specific T cells at the lymph nodes and prime antigen-specific $\mathrm{CD} 4^{+} \mathrm{T}$ cells to become activated $\mathrm{CD} 4^{+} \mathrm{T}$ cells and $\mathrm{CD} 8^{+}$ T cells to differentiate into cytotoxic T lymphocytes (CTLs) [10, 15]. Consequently, this cell-based immunity is necessary for fighting HIV infection [19, 26].

However, several studies have revealed that some DC subtypes are susceptible to HIV infection and that the infection engenders interference of the antigen-presenting function of DCs $[10,20,22,24,40]$. The modulation of DCs by HIV infection is a key aspect in viral pathogenesis. It contributes to viral evasion from immunity because the dysfunction of DC engenders some impairment effects for CTL inducement [11, 26, 34, 35]. Furthermore, progressive loss of the DC number and function during the course of HIV-1 infection are reported in [10, 11, 24]. These findings suggest that the loss of DC number and function in HIV infection might contribute to development of AIDS.

As described herein, we use a simple mathematical model to reveal how loss of the DC number and function correlate with HIV disease progression. Because the progressive decrease of DC number and function are observed [10, 11, 24], we simply assumed that the immune impairment effect increases over the HIV infection. Herein, the disease progression dynamics of our model are classifiable into four processes by virological properties. It is particularly interesting a typical disease progression presents a so-called "Risky threshold" and an "Immunodeficiency threshold" on the impairment rate. Between these thresholds, infected individuals develop to the immunodeficiency phase stochastically depending on their virological and immunological states. Furthermore, to test our theoretical framework, we investigated the existence of these thresholds in 10 patients using previously estimated parameters in $[8,23,29,37]$. We confirmed that all patients take the typical disease progression and that the risky and immunodeficiency thresholds are distributed in 


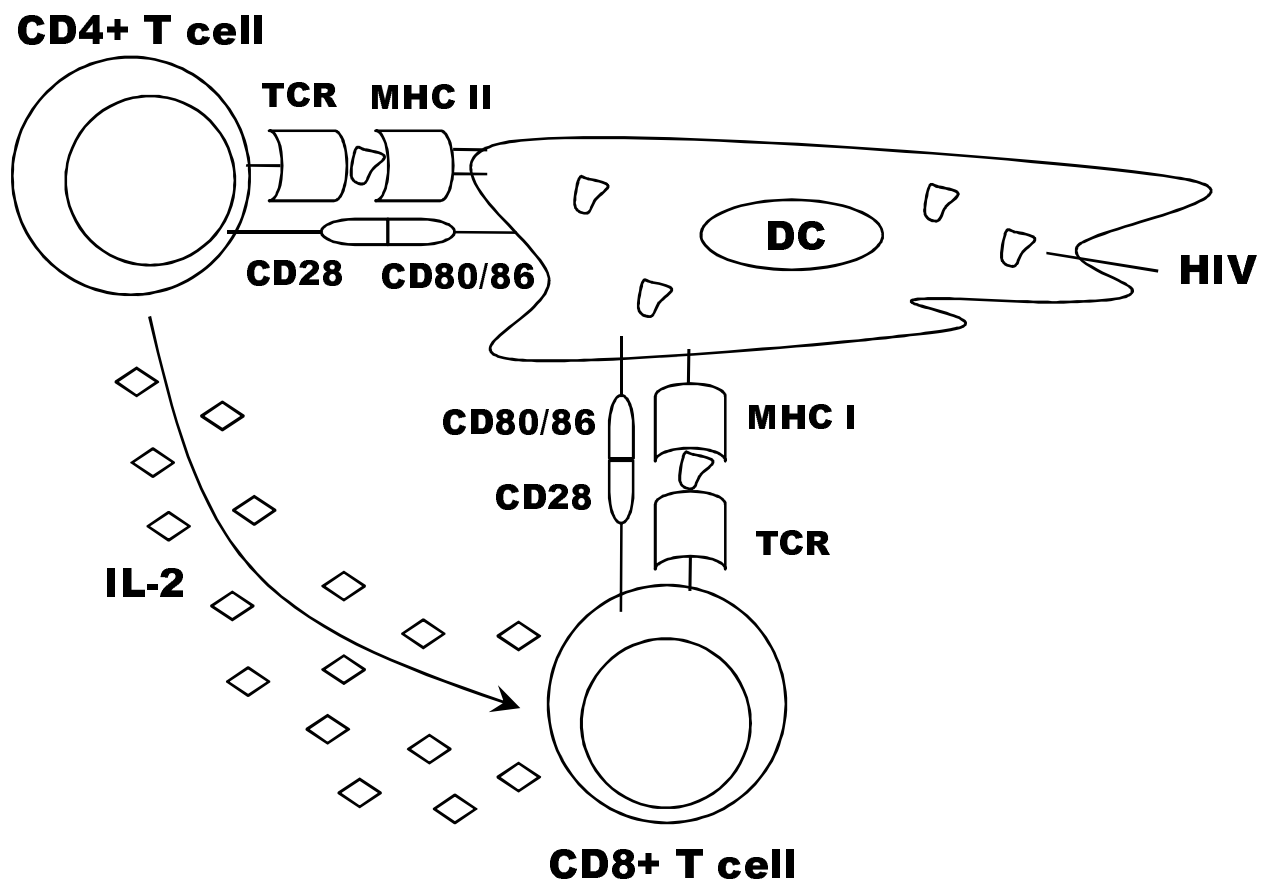

Figure 1: Summary of function of dendritic cell and its disturbance by HIV infection: after encountering HIV in the peripheral blood, DCs mature and travel to lymphoid organs. The mature DCs present HIV on MHC class II to prime CD4 ${ }^{+} \mathrm{T}$ cells to become activated $\mathrm{CD}^{+}{ }^{+} \mathrm{T}$ cells, which can secrete helper signals (e.g. IL-2). Furthermore, the DCs crosspresent HIV on MHC class I to prime $\mathrm{CD} 8^{+} \mathrm{T}$ cells. By receiving an antigen signal from the DCs and a helper signal from activated $\mathrm{CD} 4^{+} \mathrm{T}$ cells, $\mathrm{CD} 8^{+} \mathrm{T}$ cells expand and differentiate into CTLs to kill HIV infected cells. On the other hand, some DC subtypes are reportedly susceptible to HIV infection. The infection of DCs, for example, reduces expression of the co-stimulatory molecules CD80 and CD86 on the DC surface, which are required for antigen presentation to $\mathrm{T}$ cells (to combine with CD28 on the T cell surface). Consequently, the downregulated expression of CD80 and CD86 engenders subsequent impaired activation of $\mathrm{CD} 8^{+} \mathrm{T}$ cells. 
similar ranges, irrespective of virological and immunological differences.

Results of our study suggest that a decrease of the immune impairment rate (e.g., we might recover the DC function and increase the DC number by DC immunotherapy, which is a current HIV clinical trial [7, 14, 22, 34, 40]) might induce immune responses and delay the disease progression.

\section{Materials and Methods}

For this study, we use a simple mathematical model and previously estimated parameters $[8,23,29,37]$. Here we explain our modeling approach, basic assumptions, and scenario in detail.

\subsection{HIV and immune cells dynamics}

We describe interactions between HIV and its specific immune responses in the context of immune impairment effects caused by the viral infection. Here, as described in $[5,37]$, we only consider activated $\mathrm{CD} 4^{+} \mathrm{T}$ cells $(x)$ as target cells for HIV because other cell populations bearing CD4 molecules such as naive CD4 ${ }^{+} \mathrm{T}$ cells, resting $\mathrm{CD}^{+} \mathrm{T}$ cells, and macrophages are less commonly infected by HIV than activated $\mathrm{CD}^{+} \mathrm{T}$ cells are $[5,15,37]$. We assume that activated $\mathrm{CD} 4^{+} \mathrm{T}$ cells are produced at a rate of $\lambda$ cells day ${ }^{-1}$, decay at a rate $d$ day $^{-1}[5,37]$, and can become infected at a rate that is proportional to the number of infected $\mathrm{CD}^{+} \mathrm{T}$ cells $(y)$ with a transmission rate constant $\beta$ day $^{-1}$ cell $^{-1}[18,41]$. The infected $\mathrm{CD} 4^{+} \mathrm{T}$ cells are assumed to decay at the rate of $a$ day $^{-1}$. To suppress HIV replication, our immune system induces CTL responses through interactions with DCs (see Fig. 1). Here we consider that the CTL responses $(z)$ include both CTL effector and memory (CTL naives are assumed to be described by an initial value of $z$ ). The CTL responses eliminate infected $\mathrm{CD} 4^{+} \mathrm{T}$ cells at a rate that is proportional to the number of CTLs with a killing rate constant $p$ day $^{-1}$ cell $^{-1}$ and decay at a rate of $b$ day $^{-1}[18,41]$. We simply assume that proliferation of CTLs (i.e., expansion and differentiation) requires both antigen and a helper signal and that its rate is $c$ day $^{-1}$ cell ${ }^{-2}$. This implies that, in the absence of any immune impairment effects, proliferation is described by cxyz $[1,32,41]$. However, in the presence of immune impairment effects caused by HIV infection, CTL proliferation is reduced (a detailed explanation is provided later). Therefore, we model immune impairment effects as cxyz/(1+عy). The impairment effect depends on the number of infected $\mathrm{CD} 4^{+} \mathrm{T}$ cells; $\varepsilon$ represents the immune impairment rate (unit is cell ${ }^{-1}$ ). 


\subsection{Mathematical model}

We extend the standard virus-immune model including the effect of immune impairment caused by HIV infection [28]. Our mathematical model can be represented by the following equations:

$$
\begin{aligned}
x^{\prime} & =\lambda-d x-\beta x y, \\
y^{\prime} & =\beta x y-a y-p y z, \\
z^{\prime} & =\frac{c x y z}{1+\varepsilon y}-b z .
\end{aligned}
$$

Because free virus populations decay at much higher rates than the other $\mathrm{T}$ cells, we can assume that the virus population is in a quasi-steady state [38], and that model (1) [18, 41] neglects the free virus dynamics.

\subsection{Estimation of parameter values}

Baseline values of model parameters for simulations and their respective ranges for sensitivity analysis are presented in Table 1 . These parameters are based on previously estimated parameters in [8, 23, 29, 37].

In [37], M. A. Stafford et al. estimated parameter values of $\lambda, d, \beta$, and $a$ using 10 patients' virus concentration data. They assumed the ratio of activated $\mathrm{CD} 4^{+}$ $\mathrm{T}$ cells before infection at $1 \%$ of the $\mathrm{CD}^{+} \mathrm{T}$ cells in peripheral blood. Here we also use this assumption. Therefore, before infection, we fix the number of activated $\mathrm{CD}^{+} \mathrm{T}$ cells at 10 cells per $\mu l$ of peripheral blood (i.e., $x_{h}=10$ at the healthy state $E_{h}$ : see Results) because a healthy human adult has about $1000 \mathrm{CD} 4^{+} \mathrm{T}$ cells $\mu l^{-1}[19,28]$. Herein, we can estimate that the proliferation rate of activated CD4 ${ }^{+}$ $\mathrm{T}$ cells is $\lambda=d x_{h}$ for each patient. Furthermore, we calculate the infection rate of $\mathrm{CD}^{+} \mathrm{T}$ cells $\beta$ as $\pi k / c$ using original estimated parameter values $\pi, k$ and $c$ in [37] because we neglect the free virus dynamics. Furthermore, we use the estimated values of the decay rate of activated $\mathrm{CD} 4^{+} \mathrm{T}$ cells $d$ directly, along with those of infected $\mathrm{CD}^{+} \mathrm{T}$ cells $a$.

Next, we estimate immunological parameter values (which are not based on virus concentration data of the 10 patients). The killing rate of infected $\mathrm{CD} 4^{+} \mathrm{T}$ cells by CTLs is estimated at $p=0.016$ cell $^{-1}$ day $^{-1}$ in [23]. This parameter does not affect our main results at all (see Results). Therefore, we simply assume that the killing rate is the same in all patients and use it. In [29], the mean life-span of CTL effector is estimated as 80 days (i.e., the decay rate is $b=0.0125$ day $^{-1}$ ). We use the same value for this study. Here, to investigate the sensitivity of the parameter (or a difference among patients), we assume that the CTL decay rate $b$ 
ranges from 0.0025 day $^{-1}$ (i.e., one-fifth of the value) to 0.025 day $^{-1}$ (i.e., double the value) (because we consider both the CTL effector and memory, we investigate the long-life range of the CTLs). Furthermore, in [8], a doubling time of CTLs is estimated as about 18 hours. If we assume that $\mathrm{CD} 4^{+} \mathrm{T}$ cells and infected $\mathrm{CD} 4^{+} \mathrm{T}$ cells remain at steady state $E_{u}$ (at which the virus load equilibrates with no immune response: see Results) and the impairment effect is sufficiently small (i.e., $\varepsilon \approx 0$ ) during a primary expansion and differentiation of CTLs in the acute infection, we can roughly estimate the CTL proliferation rate as $c=2.13$ cell $^{-2}$ day $^{-1}$ (we used the average value $x_{u}=1.81$ and $y_{u}=0.24$ for 10 patients at $E_{u}$ and the mean decay rate of CTL $b=0.0125$ day $^{-1}$ ). To investigate a parameter's sensitivity, we assume that the CTL proliferation rate $c$ ranges from 0.426 cell $^{-2}$ day $^{-1}$ (i.e., a one-fifth of the value) to $4.26 \mathrm{cell}^{-2} \mathrm{day}^{-1}$ (i.e., double the value). Moreover, because we are interested in progressive immune impairment effects and their threshold phenomena (see Results), we need not estimate $\varepsilon$ and set it as a free parameter.

In Appendix B, we investigate how our mathematical model fits the 10 patients' virus concentration data using our baseline parameter values in Table 1.

\subsection{Immune impairment effect over HIV infection}

Several DC populations are targets for HIV [15, 22, 40]. Consequently, the ability of DCs (e.g. antigen presentation) to stimulate $\mathrm{T}$ cell proliferation is impaired because of the depletion and dysfunction of DC (e.g. downregulation of CD80/86 and MHC II expression) [10, 11, 20, 22, 24, 30]. The evidence comes from observations that DCs express CD4 and chemokine receptors and are susceptible to HIV infection in vitro $[30,40]$. Progressive depletion of DC and its functional defects, which would in turn impair generation of CTLs in patients with HIV-1 infection are observed $[10,11,24]$ and the reduction of DC number and function is particularly marked in patients with AIDS compared with asymptomatic subjects $[24,30]$. Therefore, the progressive change of DC might contribute to the immunodeficiency associated with HIV-1 disease $[10,11,20,22,24,30,40]$. To elucidate how the progressive depletion and functional defect of DCs affect disease symptoms, we simply assume that the immune impairment rate $(\varepsilon)$ gradually increases. Therefore, CTL proliferation decreases over the HIV infection. We need not consider dynamics of DC population directly to capture the essence of the progressive immune impairment effect over HIV infection. 


\section{Results}

We investigate how the loss of DC number and function (i.e. increase of immune impairment rate) correlates with HIV disease progression and demonstrate the existence of various thresholds that can determine patients' symptoms.

\subsection{Steady states during HIV infection}

We explain the virological and immunological meanings of possible steady states of our model (1).

In a healthy human, only activated $\mathrm{CD} 4^{+} \mathrm{T}$ cells attain an equilibrium level of $x_{h}$. We designate this homeostatic equilibrium $E_{h}=\left(x_{h}, 0,0\right)$ as a "healthy state" $[28]$.

After infection of HIV, if the basic reproduction number of the virus, $R_{0}=$ $\lambda \beta / a d$, is greater than 1 , then infected $\mathrm{CD} 4^{+} \mathrm{T}$ cells increase initially to a high level and subsequently converge to an equilibrium value $y_{u}$. Furthermore, activated CD4 ${ }^{+}$ $\mathrm{T}$ cells attain an equilibrium level of $x_{u}$. This equilibrium $E_{u}=\left(x_{u}, y_{u}, 0\right)$ represents a state in which the virus load of HIV is balanced with no immune response because of a shortage of activated $\mathrm{CD} 4^{+} \mathrm{T}$ cells during a primary phase of $\mathrm{HIV}$ infection [28]. In this case, we designate the steady state $E_{u}$ as a "shortage state".

In addition, at the end of the primary phase, if CTL responses are induced, then the infected cells are regulated by them for a long time at some steady state $[19,26]$. Actually our model has two possible interior equilibria $-E_{c}^{-}=\left(x_{c}^{-}, y_{c}^{-}, z_{c}^{-}\right)$and $E_{c}^{+}=\left(x_{c}^{+}, y_{c}^{+}, z_{c}^{+}\right)-$but $E_{c}^{-}$is not biologically appropriate because the equilibrium is always unstable even if it exists (see Appendix). Therefore, we consider the equilibrium $E_{c}^{+}$a "controlled state", in which effective and sustained CTLs have been established and the virus load is suppressed at a low level.

On the other hand, if CTLs are not induced at the end of the primary phase, then the infected individuals immediately develop AIDS after (or during) the acute infection [4, 6, 27]. Moreover, even if CTLs are induced, the immune responses gradually weaken and some infected individuals, in a late stage of the infection, develop AIDS [19, 26, 43]. Consequently, when a complete breakdown of the immune system occurs (implying that $z$ converges to 0 ), activated and infected CD4 ${ }^{+} \mathrm{T}$ cells also converge to the same equilibrium values of $E_{u}$. In this case, we call the steady state $E_{u}$ the "immunodeficiency state".

Here we remark that equilibrium $E_{u}$ has two meanings (the shortage state during the acute infection and the immunodeficiency state over the disease progression) in our model (1). Later in this report, we provide a detailed explanation about the 
qualitatively different properties of these two states.

\subsection{Classification of disease progression dynamics}

We assume that the basic reproduction number of HIV, $R_{0}$, is greater than 1 and that HIV establishes the infection [28]. Herein, we can divide the patterns of disease progression of our model (1) into four cases ((i)-(iv)) (Fig. 2). Because we are interested in the long-term dynamics of HIV infection, we assume that HIV and immune dynamics can be considered at a steady state after the acute infection.

We calculate the CD4 ${ }^{+} \mathrm{T}$ cell number, virus load of HIV, and CTL activity at a possible equilibrium in Fig. 2 using the average values of the parameters $\lambda, d, \beta$, and $a$ among 10 patients in Table 1 . The black and red solid lines respectively represent cell numbers at the controlled and immunodeficiency state over HIV infection (black dashed lines represent cell numbers at $E_{c}^{-}$).

(i) When viral infectivity (i.e., infection rate of HIV: $\beta$ ) is remarkably large, in the sense that $\lambda c / b<\beta$, our immune system does not establish CTL responses in spite of a very high viral load (Fig. 2(i): we used 100-fold viral infectivity $\beta$ to satisfy with $\lambda c / b<\beta)$. Too many $\mathrm{CD}^{+} \mathrm{T}$ cells are destroyed during the acute infection. Therefore, infected individuals immediately develop immunodeficiency without an asymptomatic phase $[4,6,27]$. An example for rapid disease progression is observed in experimental SIV/SHIV infection $[4,21]$.

On the other hand, when the viral infectivity is small, in the sense that $\lambda c / b>\beta$, our immune system can establish sustained CTL responses. In this case, the disease progression dynamics is determined by the following cytopathogenic thresholds:

$$
a_{-}=\frac{\lambda \beta}{d}-\frac{\beta \sqrt{\lambda c b \beta}}{c d}, \quad \bar{a}=\frac{\lambda \beta}{d}-\frac{b \beta^{2}}{c d} .
$$

Therefore, (ii) when the cytopathogenicity (i.e., death rate of infected CD4 ${ }^{+} \mathrm{T}$ cells) is small, in the sense that $0<a<a_{-}$, the sustained CTLs suppress HIV replication for a long time after the acute infection (Fig. 2(ii)). However, as the immune impairment rate increases, the CTL responses weaken gradually and the infected individuals eventually develop AIDS because of depletion of CD4 ${ }^{+} \mathrm{T}$ cells. This progressive immune decline corresponds to the typical disease progression of HIV infection [19]. Over the disease progression, we have the following two immune impairment thresholds which can determine patients' symptoms:

$$
\bar{\varepsilon}=\frac{a c}{b \beta}+\frac{a \beta}{a d-\lambda \beta}, \quad \varepsilon_{-}=\frac{(\sqrt{b \beta}-\sqrt{\lambda c})^{2}}{b d} .
$$



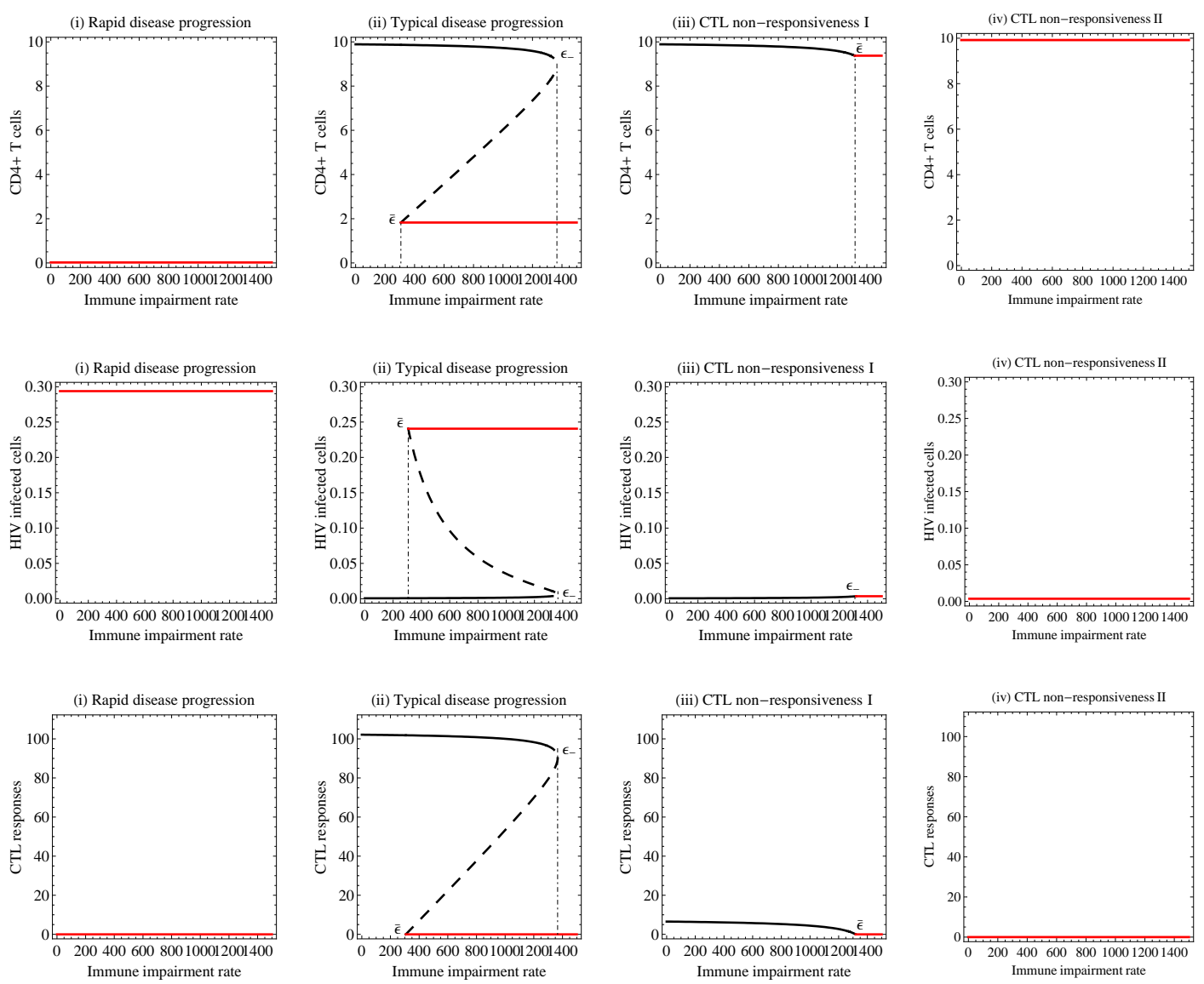

Figure 2: Classification of disease progression dynamics: the black and red solid lines respectively represent each cell number at the controlled and immunodeficiency state over HIV infection (black dashed lines represent each cell number at $E_{c}^{-}$). (i) When the infectivity is large, our immune system does not establish CTL responses in spite of the high virus load because of the depletion of $\mathrm{CD} 4^{+} \mathrm{T}$ cells. This case corresponds to rapid disease progression of SIV/SHIV infection. On the other hand, when a viral infectivity is small, our immune system can establish sustained CTL responses. Additionally, (ii) if the cytopathogenicity is small (i.e. $0<a<a_{-}$), then the sustained CTLs suppress HIV replication for a long time. However, the CTL responses decrease gradually and infected individuals come to risk developing AIDS if the impairment rate exceeds the risky threshold (i.e. $\bar{\varepsilon}<\varepsilon$ ). They always develop AIDS if the rate exceeds the immunodeficiency threshold (i.e. $\varepsilon_{-}<\varepsilon$ ). This case corresponds to typical disease progression of HIV infection. Furthermore, regarding (iii) and (iv), if cytopathogenicity is not small (i.e., $a_{-}<a<\bar{a}$ or $\left.\bar{a}<a<a_{\max }\right)$, then CTL responses are slight or nonexistent because of a lack of antigen signals. These cases correspond to CTL non-responsiveness, which differs from immunodeficiency. This phenomenon might be observed in some viral infection with a high degree of cytopathogenicity, but is inapplicable to HIV infection. 
Until the impairment rate exceeds $\bar{\varepsilon}$, CTLs control the virus load at a very low level. However, if the impairment rate exceeds this threshold value, the patients risk development of AIDS because both the controlled and immunodeficiency states can be stable simultaneously (see Appendix). Stochastic perturbation in patients caused by virological and immunological events might change the patients' state from the controlled state to the immunodeficiency state [17]. Here we call $\bar{\varepsilon}$ the "Risky threshold". By evaluating the eigenvalues at $E_{u}$ and $E_{c}^{-}$in detail, we can show that the risky threshold corresponds to a transcritical bifurcation point (see Appendix). Moreover, once the impairment rate becomes greater than $\varepsilon_{-}$, our immune system collapses completely, the CTL responses become inactivated, and the patients always develops AIDS. This is true because the controlled equilibrium is degenerated, but the immunodeficiency equilibrium remains stable. We designate $\varepsilon_{-}$as the "Immunodeficiency threshold". By evaluating the eigenvalues at $E_{c}^{-}$and $E_{c}^{+}$in detail, we can show that the immunodeficiency threshold corresponds to a saddle-node bifurcation point (see Appendix). We investigate this typical disease progression in detail later.

Furthermore, when cytopathogenicity is not small ((iii) $a_{-}<a<\bar{a}$ or (iv) $\left.\bar{a}<a<a_{\max }\right)$, CTL responses are slight or nonexistent because of the lack of antigen signals (see Figs. 2(iii) and 2(iv): we used adjusted cytopathogenicities $a$, which can be satisfied respectively with $a_{-}<a<\bar{a}$ and $\left.\bar{a}<a<a_{\max }\right)$. Here we mention that $a_{\max }=\lambda \beta / d$ is derived from the assumption that $R_{0}>1$. Actually, even if CTL responses are not induced, the viral load remains at a very low level in both cases (therefore, a slight immune response eventually becomes inactivated in case (iii)). These phenomena are considered as CTL non-responsiveness, which differs from the immunodeficiency associated with HIV infection [41] (AIDS is characterized by a high virus load of HIV and CD4 ${ }^{+} \mathrm{T}$ cell depletion [19]). The CTL non-responsiveness might be observed in a viral infection with a high degree of cytopathogenicity, but it is inapplicable to HIV infection.

\subsection{A progressive risk of immunodeficiency in HIV infection}

We investigate a progressive risk of immunodeficiency by evaluating a basin of attraction of the immunodeficiency state and provide a detailed explanation of the typical disease progression. Here we also assume that the parameters $\lambda, d, \beta$, and $a$ are the average values of 10 patients in Table 1 . Then the risky and immunodeficiency thresholds to $\bar{\varepsilon}=303.63$ and $\varepsilon_{-}=1366.45$ are estimated respectively.

In Fig. 3, we plot a basin of attraction (i.e. absorbing region) of immunodeficiency state $E_{u}$ on $x-z$ space with $y=y_{u}=0.244$ as a red region and call it the 
(i) $\varepsilon=200$

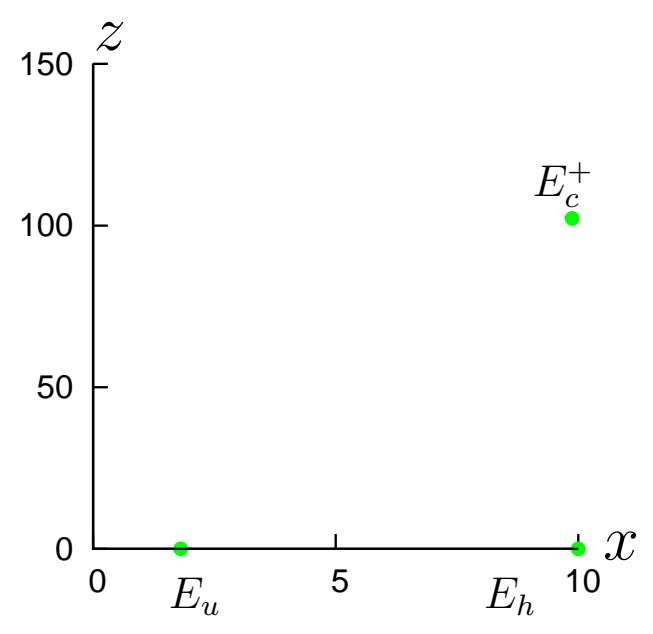

(iii) $\varepsilon=800$

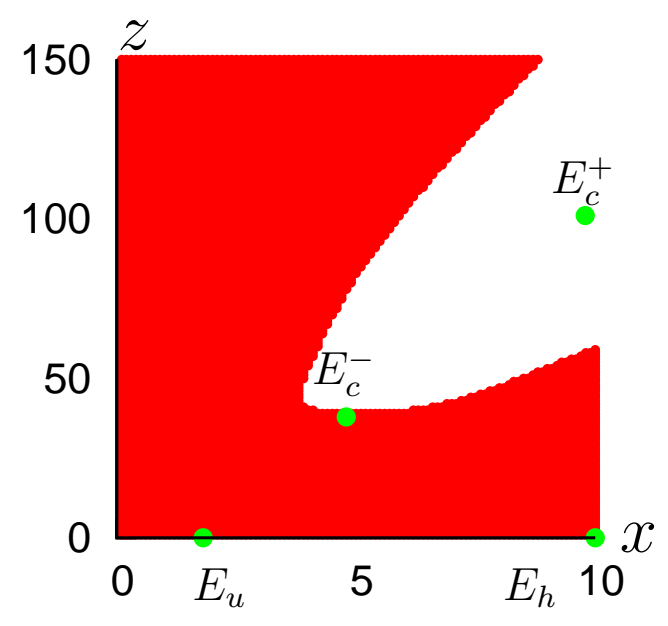

(ii) $\varepsilon=400$

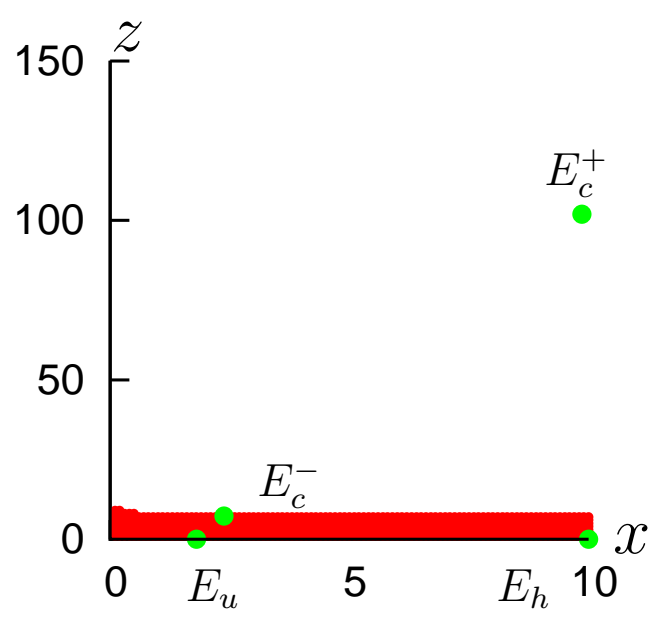

(iv) $\varepsilon=1600$

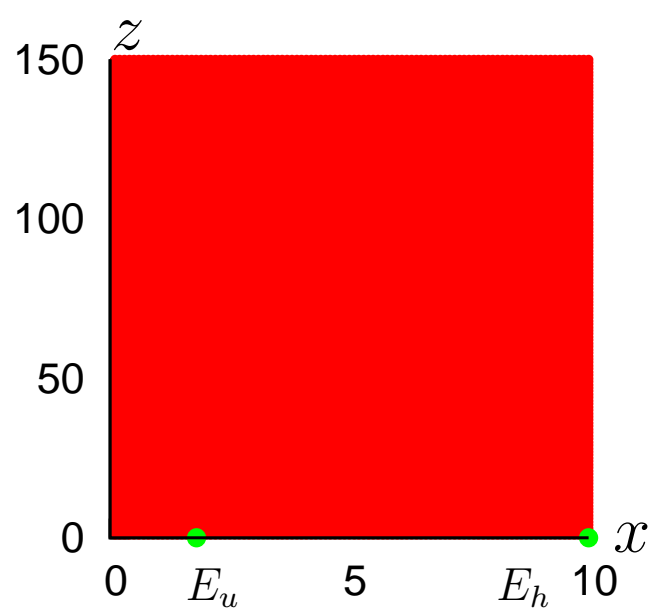

Figure 3: Risky zone of immunodeficiency: we plot a basin of attraction of $E_{u}$ as a red region and call it a "Risky zone". For (i), until the immune impairment rate $(\varepsilon)$ exceeds the risky threshold $(\bar{\varepsilon})$, only $E_{c}^{+}$is stable. On the other hand, with (ii), when the impairment rate exceeds the risky threshold, $E_{c}^{-}$is generated into $\mathbb{R}_{+}^{3}$ via a transcritical bifurcation with $E_{u}$ and the risky zone emerges (i.e., both $E_{c}^{+}$and $E_{u}$ become stable simultaneously). The stable manifold of $E_{c}^{-}$forms a boundary surface of the risky zone. Furthermore, in (iii), the risky zone expands during the impairment rate increases until the immunodeficiency threshold $\left(\varepsilon_{-}\right)$. However, in (iv), once the impairment rate becomes greater than the immunodeficiency threshold, $E_{c}^{ \pm}$are degenerated via a saddle-node bifurcation and the full space becomes the risky zone (i.e. only $E_{u}$ is stable). Here the risky and immunodeficiency thresholds to $\bar{\varepsilon}=303.63$ and $\varepsilon_{-}=1366.45$ are estimated respectively. 
"Risky zone". Therefore, if their virological and immunological states are located in the risky zone, HIV patients eventually develop immunodeficiency (i.e., all orbits converge to $E_{u}$ if their initial values are chosen from the risky zone).

We assume that the respective activated and infected $\mathrm{CD} 4^{+} \mathrm{T}$ cell dynamics have already converged to the shortage state $E_{u}$ during primary expansion and differentiation of CTLs in the acute infection. The immune impairment rate is low at the beginning of the infection. Therefore, sustained CTL responses are established; therefore, the viral replication is suppressed at a low level in the stable controlled state $E_{c}^{+}$after the CTL naives begin to expand and differentiate (Fig. 3(i)). Consequently, the virus load of HIV equilibrates and remains at a virological set point immediately after the acute infection $[19,26]$. Here we remark that the shortage state $E_{u}$ is stable in $x-y$ space but is unstable in all space if $\varepsilon$ is small, which implies that convergent steady state of model (1) always transfers from $E_{u}$ to $E_{c}^{+}$if $z$ becomes positive. Furthermore, even if the immune impairment rate increases, the viral replication is well controlled by CTL responses at $E_{c}^{+}$until the rate exceeds the risky threshold (i.e., $0<\varepsilon<\bar{\varepsilon}$ ). Actually, the immune suppression is robust for any stochastic perturbation because only $E_{c}^{+}$is stable.

On the other hand, when the impairment rate becomes greater than the risky threshold (i.e., $\bar{\varepsilon}<\varepsilon$ ), the shortage state becomes the immunodeficiency state (i.e., $E_{u}$ becomes stable in all space, which differs qualitatively from the shortage state) and the risky zone emerges (Fig. 3(ii)). Therefore, patients have a risk of development of immunodeficiency because stochastic perturbations caused by virological and immunological events, such as mutational changes of viral epitopes and their specific immune responses [25, 26, 28], a switch of coreceptor from CCR5 to CXCR4 [19], and an emergence of drug resistance [33] might drive patients from the controlled state $E_{c}^{+}$to the risky zone [17]. Furthermore, the risky zone expands gradually (Fig. 3(iii)) as the impairment rate increases until the immunodeficiency threshold $\left(\varepsilon<\varepsilon_{-}\right)$is reached. Therefore, the risk is increased progressively. Consequently, the patients become sensitive to these stochastic perturbations.

However, once the impairment rate exceeds the immunodeficiency threshold $\left(\varepsilon_{-}<\varepsilon\right)$, the risky zone expands into total space and the patients always develop AIDS (Fig. 3(iv)) because the immunodeficiency state $E_{u}$ becomes a unique stable steady state, implying that a complete breakdown of the immune system occurs and that CTL responses no longer become re-activated.

Consequently, even if viral replication can be suppressed by CTLs at the virological set point immediately after the acute infection (Fig. 3(i)), as the immune impairment rate increases, patients tend to confront the risk of development of 
immunodeficiency (Fig. 3(ii)); that risk gradually increases (Fig. 3(iii)). They eventually develop AIDS (Fig. 3(iv)).

\subsection{Immune impairment thresholds in patients}

Using our baseline parameter values and ranges in Table 1, we investigate the existence of risky and immunodeficiency thresholds among 10 patients and their crosssectional distributions.

For an arbitrary value between the CTL proliferation (decay) rate $c(b)$ in Table 1 , the viral infectivity and the cytopathogenicity of each patient are satisfied respectively with $\lambda c / b>\beta$ and $0<a<a_{-}$. For that reason, all patients take on typical disease progression, which implies that all patients have risky and immunodeficiency thresholds. We perform sensitivity analysis in Fig. 4 for variations in the immune impairment thresholds among 10 patients with respective ranges of (i) the proliferation rate and (ii) the decay rate of CTLs in Table 1 because these parameter values are not estimated from virus concentration data of the patients in [37] (we remark that, because these thresholds are independent of the killing rate of infected $\mathrm{CD}^{+} \mathrm{T}$ cells $p$, we need not investigate their range). The colored triangle $(\boldsymbol{\Delta})$ represents threshold values with estimated parameter values. The colorless square $(\square)$ and circle $(\bigcirc)$ respectively represent the maximum and minimum threshold values within the ranges.

Figure 4 portrays that the distributions of risky and immunodeficiency thresholds (particularly, the maximum values of risky thresholds) are more sensitive to the CTL decay rate than to its proliferation rate. However, risky and immunodeficiency thresholds among 10 patients seem to be distributed in close ranges in Fig. 4 , irrespective of virological and immunological differences (threshold values with the estimated parameter values $(\boldsymbol{\Delta})$ are distributed almost uniformly), which might imply that all patients tend to have a risk of development of AIDS with a close timing and that they eventually develop AIDS in a close range of the impairment rate (actually, in terms of our mathematical model, the length of asymptomatic phase is characterized by the wide shape of its distribution and the development is stochastic once the impairment rate exceeds the risky threshold [17]). These cross-sectional studies based on our mathematical model predict the possibility of the existence of immune impairment thresholds in HIV infection. Therefore, our theoretical framework of immune impairment effects over HIV infection might provide clear insight into the time when patients develop AIDS (i.e., a prediction of AIDS development). 
(i) Risky threshold : $\bar{\epsilon}$ (sensitivity of $c$ )

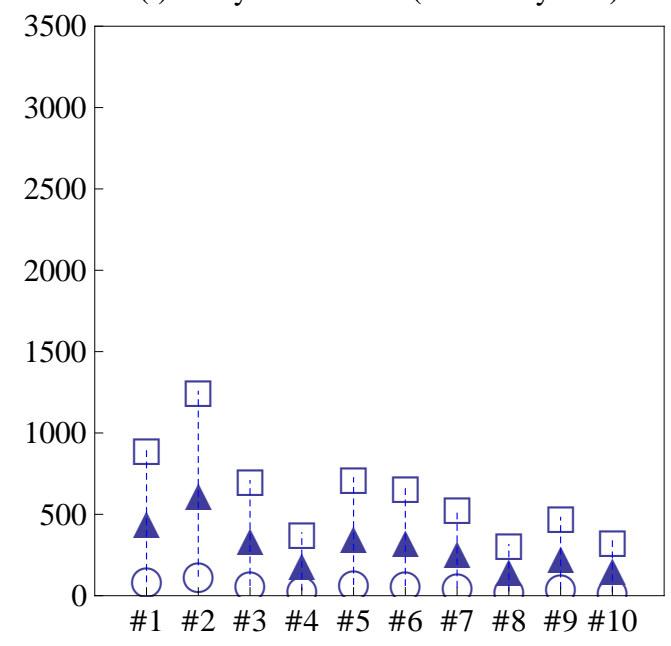

(i) Immunodeficiency threshold : $\epsilon_{-}$(sensitivity of $c$ )

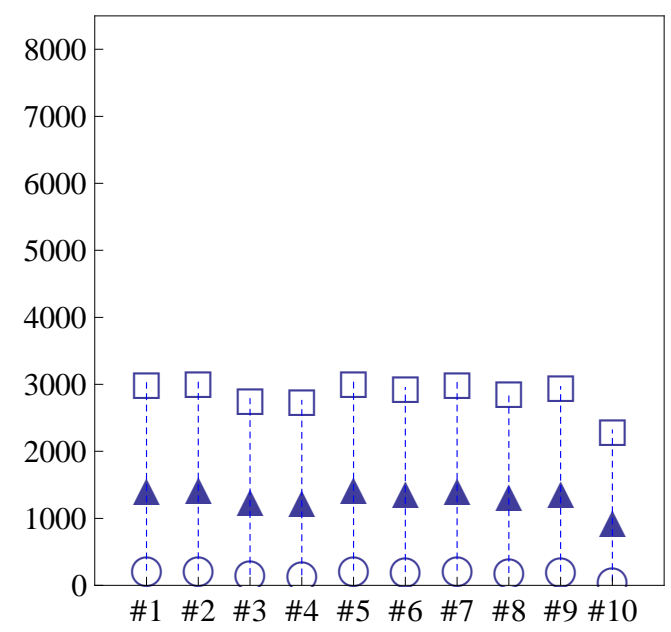

(ii) Risky threshold : $\bar{\epsilon}$ (sensitivity of $b$ )

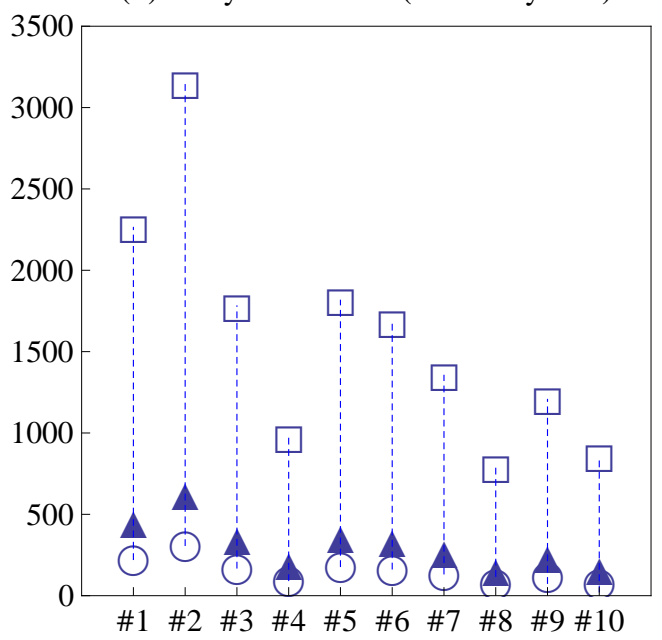

(ii) Immunodeficiency threshold : $\epsilon_{-}$(sensitivity of $b$ )

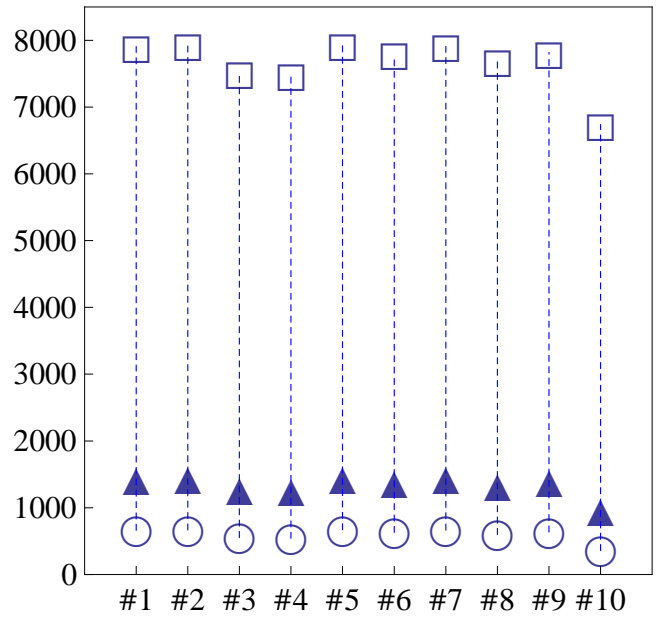

Figure 4: Distribution of risky and immunodeficiency thresholds among 10 patients: we perform sensitivity analysis for variations in the immune impairment thresholds among 10 patients with respective ranges of (i) the proliferation rate and (ii) the decay rate of CTLs in Table 1. The colored triangle $(\boldsymbol{\Delta})$ represents threshold values with the estimated parameter values. The colorless square $(\square)$ and circle $(\bigcirc)$ respectively represent the maximum and minimum threshold values within the ranges. We confirm that all patients have their risky and immunodeficiency thresholds in close ranges, irrespective of virological and immunological differences. These crosssectional studies based on our mathematical model predict the possible existence of the immune impairment thresholds in HIV infection. 


\section{Discussion}

The onset of AIDS is characterized by a breakdown of the immune system after a long asymptomatic period $[19,26,43]$. The mechanistic basis of this disease progression has remained obscure, but many researchers have sought to explain it. For example, in early models of HIV infection [28], an explosion in the virus load caused by increased HIV variants' diversity explains the immune system collapse. In [41], the CTL exhaustion induced by an evolutionary increase of viral infectivity accounts for immunodeficiency. Moreover, in [13], the functional deterioration of $\mathrm{T}$ and $\mathrm{B}$ cells caused by accumulations of deleterious mutations is considered as a reason for development of AIDS. Consequently, to date, several approaches have been proposed to explain the progression of HIV infection to AIDS [2, 9, 13, 15, 16, 28, 41].

This paper presents discussion of an immune impairment effect caused by the depletion and dysfunction of DC on HIV disease progression. Because the progressive decrease of DC number and function during the course of HIV-1 infection is observed [10, 11, 24], we simply assumed that the immune impairment rate $(\varepsilon)$ increases over the HIV infection instead of considering the population dynamics of DCs directly (in contrast, in [15], to investigate the dual role of DCs (i.e. transmission of HIV and activation of immune cell), the population dynamics of DCs are involved into their model). We also remark that the immune impairment effects included in our model (1) arose from many other sources. For example, during HIV infection, programmed death 1 (PD-1) expression is elevated on HIV-specific CD8 ${ }^{+}$ $\mathrm{T}$ cells $[12]$ and engagement of PD-1 by its ligands (PD-L) inhibits several $\mathrm{CD}^{+} \mathrm{T}$ cell functions [3] (actually, blocking the PD-1-PD-L pathway engenders increased T cell proliferation and effector cytokine production [39]). The immune impairment effect driven from the PD-1-PD-L pathway also impairs CTL responses. Therefore, the effect can be described by our mathematical model. Consequently, our model is applicable to other immune impairment phenomena in HIV infection.

Results show that the patterns of disease progression of our model (1) are divided into four cases (Fig. 2). In typical disease progression, which is characterized by a low infection rate $(\beta)$ and cytopathogenicity of $\operatorname{HIV}(a)$, we derived two immune impairment thresholds: the risky threshold $(\bar{\varepsilon})$ and the immunodeficiency threshold $\left(\varepsilon_{-}\right)$(Fig. 2(ii)). Until the immune impairment rate exceeds the risky threshold, CTLs are induced and suppress viral replication (Fig. 3(i)), but once the impairment rate becomes greater than the threshold, the infected individuals face the risk of development of immunodeficiency (Figs. 3(ii)-3(iii)). Furthermore, if the impairment rate becomes greater than the immunodeficiency threshold, then patients 
always develop AIDS (Fig. 3(iv)). Consequently, our theoretical framework can explain disease progression: many experimenters have concluded that the progressive alteration of the immune system (the depletion and dysfunction of DCs are central to many of these hypotheses) might result from the development of AIDS $[10,11,20,22,24,30,35,40]$.

The central description of the development of AIDS in our model (1) is a catastrophic change of the patients' state (e.g., immunodeficiency characterized by the CTL exhaustion proposed in [41]) (Figs. 2 and 3). The mechanistic basis of the catastrophe is derived from the bistability of controlled state $E_{c}^{+}$and immunodeficiency state $E_{u}$. Actually, in several models, a bistability of steady states has been discussed to explain, for example, the multiple virological set points [2], the explosion of virus load [9], and CTL exhaustion [41]. A readily apparent difference of our model (1) from these earlier models is the consideration of the progressive immune impairment effect which depends on the number of infected $\mathrm{CD} 4^{+} \mathrm{T}$ cells. Furthermore, we were able to estimate various thresholds related to immunodeficiency strictly. Although qualitatively similar results were obtained through more complex models $[2,9,41,42]$, which can be treated analytically only to a slight degree, one important result of our model is the clear and simple concept illustrating the risk of immunodeficiency.

We also investigated the distribution of risky and immunodeficiency thresholds among 10 patients. It is particularly interesting that we showed that all patients follow the typical disease progression and that the risky and immunodeficiency thresholds are distributed in close ranges, irrespective of virological and immunological differences (Fig. 4). Here we must mention that our baseline parameter values and ranges in Table 1 could not be justified completely because, in [37], the number of activated $\mathrm{CD} 4^{+} \mathrm{T}$ cells (10 cells per $\mu l$ of peripheral blood) and the decay rate of HIV (3 day ${ }^{-1}$ which is referred to [31]) were assumed to be the same value among 10 patients. Moreover, because no data related to the patients' immune response exist, we estimated the immunological parameters separately using previously estimated parameter values in $[8,23,29]$. Therefore, these simplifications of estimation of the parameters might engender some gap in the distribution. Nevertheless, although we must estimate the exact parameter values of the 10 patients, our cross-sectional studies imply the possible existence of immune impairment thresholds in HIV infection and validity of our theory.

In summary, our results imply that recovering DC function and increasing DC number might be effective for inducing immune response and delaying the disease progression (actually, several studies of DC immunotherapy found a transient de- 
crease of the virus load by activating $\mathrm{T}$ cell responses against HIV-1 [7, 14, 34]) because the modulation of DC engenders a decrease of the immune impairment rate $(\varepsilon)$; following such a strategy, the risky zone dwindles or disappears (see Fig. 3 ). The findings described herein present important implications for the design of therapeutic vaccines. For example, reconstitution of CD80/CD86 expression in DC, which reduces the impairment effect, might be important to consider development of therapeutic HIV-1 vaccine [22]. In addition, if the impairment rate can be quantified using some data, our theory might enable prediction of the timing of AIDS development. Consequently, although our model presents several limitations because of its simplification of estimation of the parameter and its neglect of population dynamics of DCs, our study points out that further experimental investigation of DC depletion and dysfunction in patients and quantification of the immune impairment rate will be important for understanding of HIV disease progression and for development of DC vaccines and prediction of AIDS.

\section{Appendix A: Mathematical analysis}

The model has four possible equilibria:

$$
E_{h}=\left(x_{h}, 0,0\right), \quad E_{u}=\left(x_{u}, y_{u}, 0\right), \quad E_{c}^{-}=\left(x_{c}^{-}, y_{c}^{-}, z_{c}^{-}\right), \quad E_{c}^{+}=\left(x_{c}^{+}, y_{c}^{+}, z_{c}^{+}\right) .
$$

Here $x_{h}=\lambda / d, x_{u}=a / \beta, y_{u}=\left(\lambda-d x_{u}\right) / \beta x_{u}$. Furthermore, $x_{c}^{ \pm}, y_{c}^{ \pm}, z_{c}^{ \pm}$respectively signify the roots of the following equations:

$$
\begin{aligned}
& f(x)=c d x^{2}+(b \beta-\varepsilon b d-\lambda c) x+\lambda \varepsilon b=0, \\
& g(y)=\varepsilon b \beta y^{2}+(b \beta+\varepsilon b d-\lambda c) y+b d=0, \\
& h(z)=c d p^{2} z^{2}+p\{2 a c d+\beta(b \beta-\lambda c-\varepsilon b d)\} z+a^{2} d c+a \beta(b \beta-\varepsilon b d-\lambda c)+\varepsilon \lambda \beta^{2} b=0 .
\end{aligned}
$$

The basic reproductive number for one infected cell is definable as $R_{0}=\lambda \beta / a d$, which represents the average number of cells infected by a single infected cell in an otherwise susceptible cell population $([28])$.

Clearly, $E_{h}$ always exists and $E_{u}$ exists if and only if $R_{0}>1$. Next we consider the existence condition of $E_{c}^{ \pm}$. Because the discriminants for the above three functions $f, g$, and $h$ are the same and given as $D=D_{f}=D_{g}=D_{h}=\varepsilon^{2} b^{2} d^{2}-2 b d(b \beta+$ $\lambda c) \varepsilon+(b \beta-\lambda c)^{2}$, we can obtain

$$
D>0 \Longleftrightarrow \varepsilon<\frac{(\sqrt{b \beta}-\sqrt{\lambda c})^{2}}{b d}=\varepsilon_{-} \text {or } \varepsilon>\frac{(\sqrt{b \beta}+\sqrt{\lambda c})^{2}}{b d}=\varepsilon_{+} .
$$

From $f(0)>0$ and $g(0)>0$, the following relations hold:

$$
\begin{aligned}
& \text { (A) : } \quad x_{c}^{ \pm}>0 \Longleftrightarrow D>0, \quad b \beta-\varepsilon b d-\lambda c<0, \\
& \text { (B) : } \quad y_{c}^{ \pm}>0 \Longleftrightarrow D>0, \quad b \beta+\varepsilon b d-\lambda c<0 .
\end{aligned}
$$


If $\lambda c-b \beta<0$, then we have $y_{c}^{ \pm}<0$ from the last inequality in (B), which implies that $E_{c}^{ \pm}$can not exist in $\mathbb{R}_{+}^{3}$. Hereinafter we assume that $\lambda c-b \beta>0$. We define $\varepsilon_{0}=(\lambda c-b \beta) / b d$. Then the last inequality in $(\mathrm{A})$ is satisfied for any $0<\varepsilon$ and the last inequality in (B) is satisfied for $0<\varepsilon<\varepsilon_{0}$. Because $0<\varepsilon_{-}<\varepsilon_{0}$, we have $x_{c}^{ \pm}>0$ and $y_{c}^{ \pm}>0$ iff $0<\varepsilon<\varepsilon_{-}$. For $h(z)=0$, we have two cases: (i) $h(0)<0$ and (ii) $h(0)>0$. In case (i) of $h(0)<0$, it is clear that $z_{c}^{-}<0$ and $z_{c}^{+}>0$. If (a) $a d-\lambda \beta>0$ (i.e. $R_{0}<1$ ), we can show that

$$
\begin{aligned}
\bar{\varepsilon}>\varepsilon_{+} & \Longleftrightarrow \frac{a c}{b \beta}+\frac{a \beta}{a d-\lambda \beta}>\frac{(\sqrt{b \beta}+\sqrt{\lambda c})^{2}}{b d} \\
& \Longleftrightarrow c d^{2} a^{2}-2 d \beta \sqrt{\lambda c}(\sqrt{b \beta}+\sqrt{\lambda c}) a+\lambda \beta^{2}(\sqrt{b \beta}+\sqrt{\lambda c})^{2}>0 \\
& \Longleftrightarrow\{a d \sqrt{c}-\beta \sqrt{\lambda}(\sqrt{b \beta}+\sqrt{\lambda c})\}^{2}>0 .
\end{aligned}
$$

Here, $\bar{\varepsilon}=a c / b \beta+a \beta /(a d-\lambda \beta)$, which satisfies $\left.h(0)\right|_{\varepsilon=\bar{\varepsilon}}=0$. Therefore, we can obtain that

$$
a d-\lambda \beta>0, h(0)<0 \Longleftrightarrow \varepsilon>\bar{\varepsilon}, \bar{\varepsilon}>\varepsilon_{+} .
$$

If (b) $a d-\lambda \beta<0$ (i.e., $R_{0}>1$ ), we can also show that

$$
a d-\lambda \beta<0, h(0)<0 \Longleftrightarrow \varepsilon<\bar{\varepsilon}, \bar{\varepsilon}<\varepsilon_{-} .
$$

Therefore, we can conclude that if $a d-\lambda \beta>0$, then $E_{c}^{ \pm}$can not exist in $\mathbb{R}_{+}^{3}$ (see Fig. $5(\mathrm{a}))$. If $a d-\lambda \beta<0$, then we have two intervals of $a: 0<a<\bar{a}$ and $\bar{a}<a<a_{\max }$. Here $\bar{a}$ satisfies $\bar{\varepsilon}(\bar{a})=0$. When $0<a<\bar{a}$ (i.e., $0<\bar{\varepsilon}$ ), then $E_{c}^{+}$can exist under $0<\varepsilon<\bar{\varepsilon}$ and $E_{c}^{-}$can not exist in $\mathbb{R}_{+}^{3}$ (see Fig. $5(\mathrm{~b})$ ). However, when $\bar{a}<a<a_{\max }$ (i.e., $\bar{\varepsilon}<0$ ), $E_{c}^{ \pm}$can not exist in $\mathbb{R}_{+}^{3}$ for any $\varepsilon>0$ (see Fig. $5(\mathrm{~b})$ ). For the case (ii)

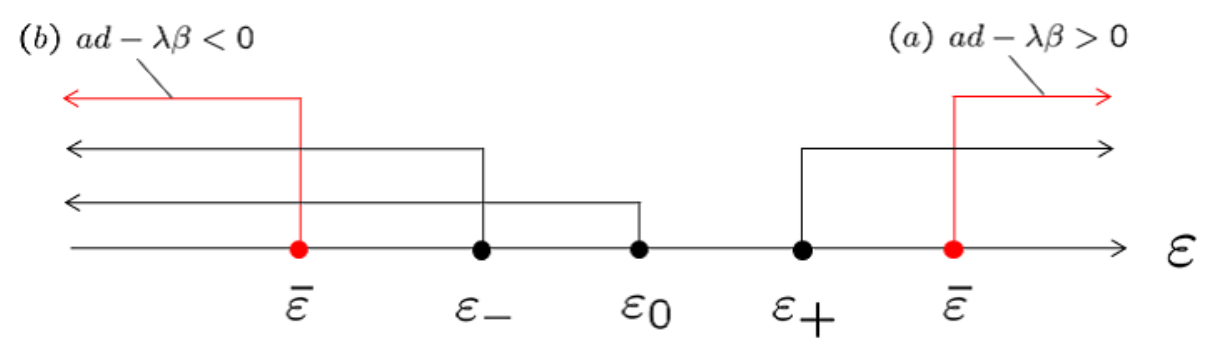

Figure 5: Graphical presentation of existence conditions for $E_{c}^{ \pm}$with $h(0)<0$

$h(0)>0$, it seems clear that $z_{c}^{ \pm}>0$ if $\beta(\lambda c+\varepsilon b d-b \beta)-2 a c d>0$ and $D>0$. The following relation holds:

$$
\beta(\lambda c+\varepsilon b d-b \beta)-2 a c d>0 \Longleftrightarrow \varepsilon>\frac{2 a c}{b \beta}+\frac{b \beta-\lambda c}{b d}=\varepsilon_{m} .
$$


Next, we let $G(a)$ denote

$$
G(a)=\bar{\varepsilon}-\varepsilon_{m}=-\frac{a c}{b \beta}+\frac{a \beta}{a d-\lambda \beta}+\frac{\lambda c-b \beta}{b d} .
$$

In fact, $G^{\prime}(a)<0, G(0)>0, \lim _{a \rightarrow \infty} G(a)=-\infty$ and $\lim _{a \rightarrow a_{\max } \pm 0} G(a)= \pm \infty$ (See Fig. 6). This implies that there exist $a_{ \pm}$such that $G\left(a_{ \pm}\right)=0$ (i.e., $\bar{\varepsilon}=\varepsilon_{m}$ ). In fact,
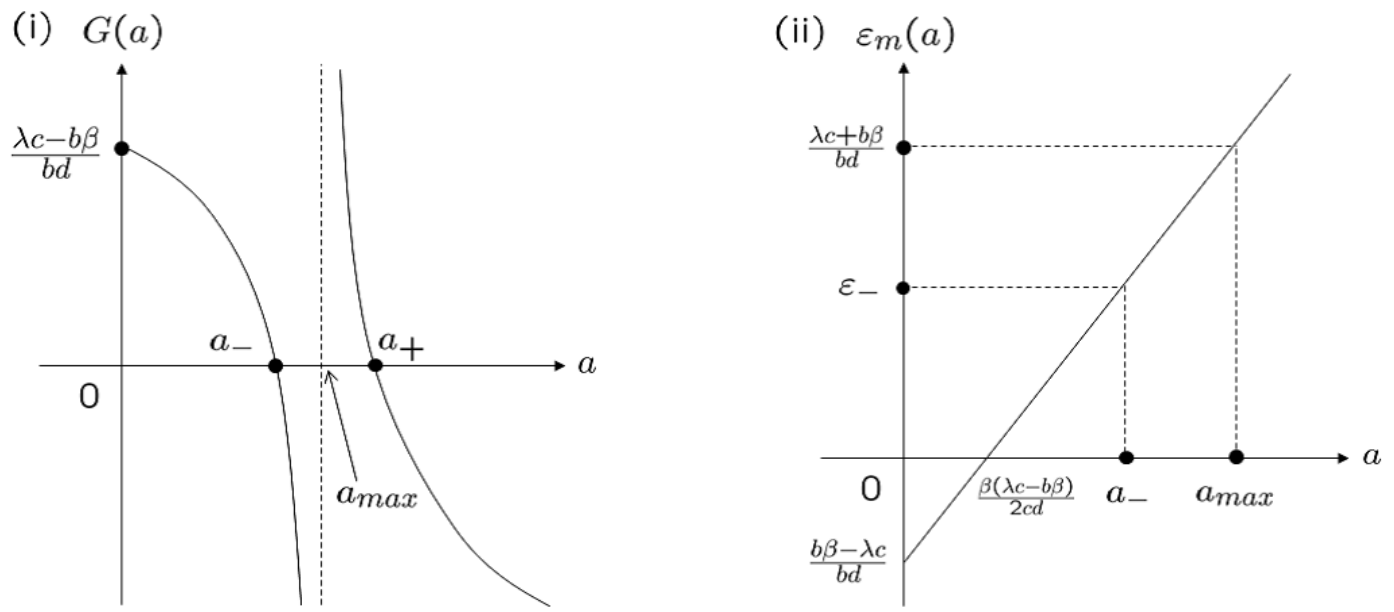

Figure 6: Graphical presentation of $G(a)$ and $\varepsilon_{m}(a)$

we have

$$
G\left(a_{ \pm}\right)=0 \Longleftrightarrow a_{ \pm}=\frac{\lambda \beta}{d} \pm \frac{\beta \sqrt{\lambda c b \beta}}{c d} .
$$

Therefore, if $0<a<a_{-}$or $a_{\max }<a<a_{+}$, then $\bar{\varepsilon}>\varepsilon_{m}$ : otherwise $\bar{\varepsilon}<\varepsilon_{m}$ (See Fig. 6(i)). In addition, for $\varepsilon_{m}$, it can be shown that $\varepsilon_{m}\left(a_{-}\right)=\varepsilon_{-}$. Consequently, if $0<a<a_{-}$, then $\varepsilon_{m}<\varepsilon_{-}$; otherwise $\varepsilon_{m}>\varepsilon_{-}$(See Fig. 6(ii)). Similarly, we can obtain the following relations for (a) $a d-\lambda \beta>0$ and (b) $a d-\lambda \beta<0$ :

$$
\begin{aligned}
& a d-\lambda \beta>0, h(0)>0 \Longleftrightarrow \varepsilon<\bar{\varepsilon}, \bar{\varepsilon}>\varepsilon_{+}, \\
& a d-\lambda \beta<0, h(0)>0 \Longleftrightarrow \varepsilon>\bar{\varepsilon}, \bar{\varepsilon}<\varepsilon_{-} .
\end{aligned}
$$

We can therefore conclude the following: if $a d-\lambda \beta>0$ (i.e., $a_{\max }<a<a_{+}$or $a_{+}<a$ ), then $E_{c}^{ \pm}$can not exist in $\mathbb{R}_{+}^{3}$ (see Fig. $7(\mathrm{a})$ ). Furthermore, if $a d-\lambda \beta<0$, then we have two intervals of $a$ : $0<a<a_{-}$and $a_{-}<a<a_{\max }$ (see Fig. 7(b)). When $0<a<a_{-}, E_{c}^{ \pm}$can exist in $\mathbb{R}_{+}^{3}$ under $\bar{\varepsilon}<\varepsilon<\varepsilon_{-}$, but when $a_{-}<a<a_{\max }$, $E_{c}^{ \pm}$can not exist in $\mathbb{R}_{+}^{3}$.

Hereinafter, we explain the stability of these equilibria in detail. From a direct calculation, the eigenvalues of $J\left(E_{h}\right)$ are $-d,-b$ and $\beta x_{h}-a$, where $J$ is the Jacobian matrix of (1), which implies that if $R_{0}<1$, then $E_{h}$ is stable; otherwise $E_{h}$ is 
(a) $a d-\lambda \beta>0$

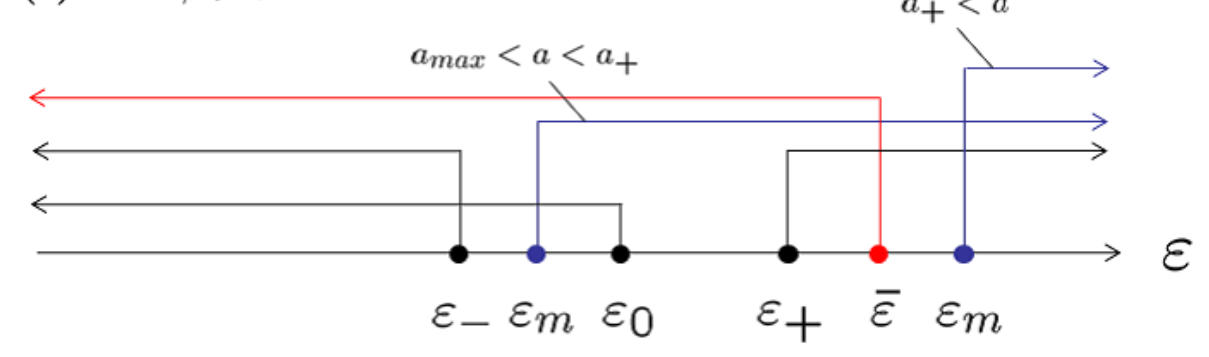

(b) $a d-\lambda \beta<0$

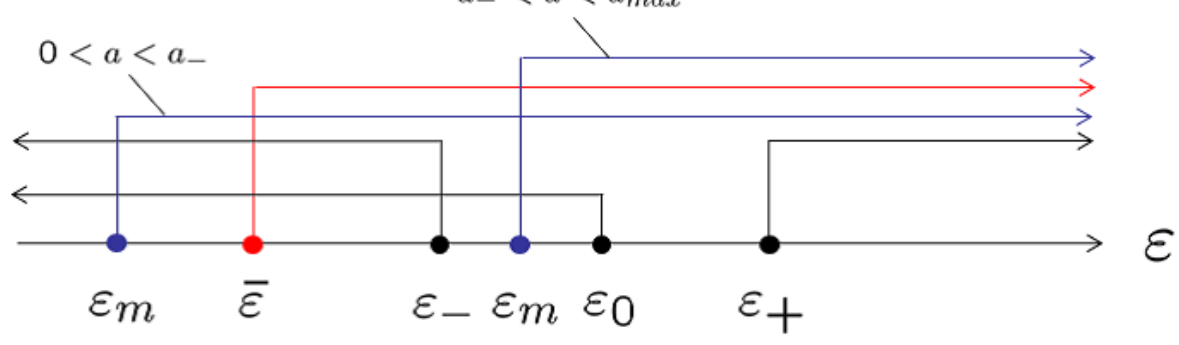

Figure 7: Graphical presentation of existence conditions for $E_{c}^{ \pm}$with $h(0)>0$

unstable. The Jacobian matrix of (1) at $E_{u}$ is

$$
J\left(E_{u}\right)=\left[\begin{array}{ccc}
-d-\beta y_{u} & -\beta x_{u} & 0 \\
\beta y_{u} & 0 & -p y_{u} \\
0 & 0 & \frac{c x_{u} y_{u}}{1+\varepsilon y_{u}}-b
\end{array}\right]
$$

We can readily confirm that the first $2 \times 2$ block is stable. Therefore, the stability of $E_{u}$ is determined by the sign of eigenvalue $c x_{u} y_{u} /\left(1+\varepsilon y_{u}\right)-b$. We can conclude that

$$
\frac{c x_{u} y_{u}}{1+\varepsilon y_{u}}-b<0 \Longleftrightarrow h(0)>0
$$

As stated earlier, $h(0)>0$ and $R_{0}>1$ are equivalent to $\bar{\varepsilon}<\varepsilon$. That is, if $\bar{\varepsilon}<\varepsilon$, then $E_{u}$ is stable; otherwise $E_{u}$ is unstable. The Jacobian matrix of (1) at $E_{c}^{ \pm}$is

$$
J\left(E_{c}^{ \pm}\right)=\left[\begin{array}{ccc}
-d-\beta y_{c}^{ \pm} & -\beta x_{c}^{ \pm} & 0 \\
\beta y_{c}^{ \pm} & 0 & -p y_{c}^{ \pm} \\
\frac{c y_{c}^{ \pm} z_{c}^{ \pm}}{1+\varepsilon y_{c}^{ \pm}} & \frac{c x_{c}^{ \pm} z_{c}^{ \pm}}{\left(1+\varepsilon y_{c}^{ \pm}\right)^{2}} & 0
\end{array}\right]
$$

The characteristic equation of $J\left(E_{c}^{ \pm}\right)$is

$$
s^{3}+a_{1} s^{2}+a_{2} s+a_{3}=0
$$

where

$$
a_{1}=d+\beta y_{c}^{ \pm}, \quad a_{2}=\beta^{2} x_{c}^{ \pm} y_{c}^{ \pm}+\frac{p c x_{c}^{ \pm} y_{c}^{ \pm} z_{c}^{ \pm}}{\left(1+\varepsilon y_{c}^{ \pm}\right)^{2}}, \quad a_{3}=\frac{p c x_{c}^{ \pm} y_{c}^{ \pm} z_{c}^{ \pm}}{1+\varepsilon y_{c}^{ \pm}}\left(\frac{d+\beta y_{c}^{ \pm}}{1+\varepsilon y_{c}^{ \pm}}-\beta y_{c}^{ \pm}\right)
$$


Here $s$ denotes the indeterminate of the polynomial. Therefore, from the RouthHurwitz criterion, all eigenvalues have negative real parts if and only if

$$
a_{1}>0, a_{3}>0, a_{1} a_{2}-a_{3}>0 .
$$

Clearly, $a_{1}>0$ and $a_{1} a_{2}-a_{3}>0$. Therefore, the stability of $E_{c}^{ \pm}$is determined by the sign of $a_{3}$. We can show

$$
a_{3}>0 \Longleftrightarrow \frac{d}{\beta \varepsilon}>y_{c}^{ \pm 2} .
$$

$y_{c}^{ \pm}$are the roots of $g(y)=0$. Therefore, we have $y_{c}^{ \pm 2}=y_{c}^{ \pm}(\lambda c-b \beta-\varepsilon b d) / \varepsilon b \beta-d / \varepsilon \beta$. This leads to

$$
\frac{d}{\beta \varepsilon}>y_{c}^{ \pm 2} \Longleftrightarrow \frac{2 b d}{\lambda c-b \beta-\varepsilon b d}>y_{c}^{ \pm}
$$

$y_{c}^{ \pm}$can be rewritten as follows:

$$
y_{c}^{ \pm}=\frac{2 b d}{\lambda c-b \beta-\varepsilon b d \pm \sqrt{(\lambda c-b \beta-\varepsilon b d)^{2}-4 \varepsilon b^{2} d \beta}}
$$

which implies that

$$
y_{c}^{+}<\frac{2 b d}{\lambda c-b \beta-\varepsilon b d}<y_{c}^{-} .
$$

Therefore, we can conclude that $E_{c}^{-}$is always unstable if it exists and $E_{c}^{+}$is always

\begin{tabular}{ccc} 
Equilibrium & Existence condition & Stability condition \\
\hline$E_{h}$ & always & $R_{0}<1$ \\
$E_{u}$ & $R_{0}>1$ & $\bar{\varepsilon}<\varepsilon$ \\
$\left(0<a<a_{-}\right)$ & \\
$E_{c}^{-}$ & $\lambda c / b>\beta, \bar{\varepsilon}<\varepsilon<\varepsilon_{-}$ & always unstable \\
$E_{c}^{+}$ & $\lambda c / b>\beta, 0 \leq \varepsilon<\varepsilon_{-}$ & always stable \\
$\left(a_{-}<a<\bar{a}\right)$ & \\
$E_{c}^{+}$ & $\lambda c / b>\beta, 0 \leq \varepsilon<\bar{\varepsilon}$ &
\end{tabular}

Table 2: Existence and stability conditions of the equilibria

stable if it exists. Consequently, we can summarize the existence and stability conditions in Table $2\left(\bar{\varepsilon}<0\right.$ if $\lambda c-b \beta<0$ and $a_{-}<\bar{a}$ if $\left.\lambda c-b \beta>0\right)$. We remark that Table 2 includes the case of $\varepsilon=0$. 


\section{Appendix B: Fit test for 10 patient data}

We check how our mathematical model fits the 10 patients' virus concentration data using our baseline parameter values in Table 1. Here, for example, we assume that, because of a time delay of immune response [19], CTL responses are induced after 30 days from the date of initial HIV infection $(z(t)=0$ for $0 \leq t<30$ and $z(30)>0$ ) and that, because of progressive depletion and dysfunction of DC $[10,11,24]$, the immune impairment accumulates with a very low rate during disease progression, $\varepsilon(t)=1.5(t-30)$, where 1.5 is the rate of average impairment per day (we do not know how the impairment rate $\varepsilon$ can be formulated but we use a simplest formulation of increasing function).

In Fig.8, we represent time-course of virus population for 2000 days for 10 patients. Here we calculate the virus population (HIV-1 RNA $\mu l^{-1}$ ) from $y(t)$ using original estimated parameter values in [37], because we neglect the free virus dynamics. That is, we define $v(t)=\pi y(t) / c$ where $\pi$ and $c$ are the notation used in [37]. The red dots during the acute infection in each panel represent the patient data which were used in parameter estimation in [37] and the black dots after the acute infection represent the data which were not used in the parameter estimation.

We found that our mathematical model and baseline parameter values fit the patient data in the short-term (red dots) but hardly fit that in the long-term (black dots). In particular, in patients 1, 2, 5, and 9, different dynamical behaviors are observed between the Stafford model and our model (we find in patients, 3, 4, 6, 7, 8, and 10, similar dynamical behaviors although our model includes the immune cell dynamics). This is because the parameter values of $\lambda, d, \beta$, and $a$ are derived from the short-term data (i.e., red dots) in [37] and these parameters might not be adequate to long-term dynamics of our mathematical model. Therefore, in order to predict the long-term virus population dynamics of the patients by our model, we have to use the parameter values estimated by using a long-term data.

\section{Acknowledgments}

The authors express their appreciation to Prof. X. Liu and Prof. W. Wang for valuable comments. We would also like to thank anonymous referees for very helpful suggestions and comments, which have improved the quality of our study. S. Iwami was supported by Research Fellowships of the Japan Society for the Promotion of Science for Young Scientists. S. Nakaoka was supported by (i) Research Fellowships of the Japan Society for the Promotion of Science for Young Scientists and (ii) the 

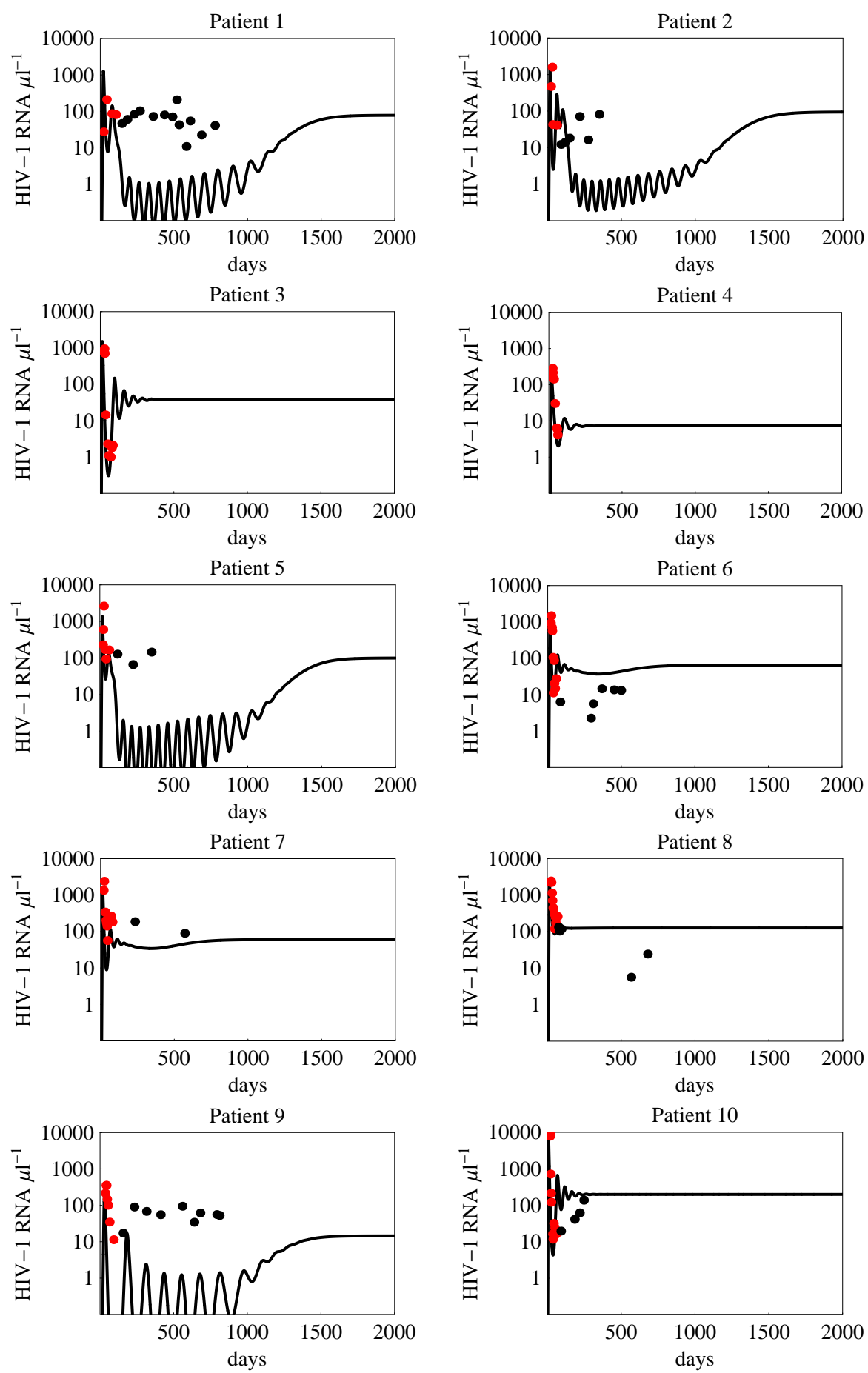

Figure 8: Fit test for 10 patient data: we investigate how our mathematical model fits the 10 patients' virus concentration data using our baseline parameter values in Table 1. Both the red and black dots represent patient data but only the red dots were used in parameter estimation in [37]. Here we calculate the virus population (HIV-1 RNA $\mu l^{-1}$ ) from $y(t)$ using original estimated parameter values in [37] (we define $v(t)=\pi y(t) / c$ where $\pi$ and $c$ are the notation used in [37]). 
Sasakawa Scientific Research Grant from The Japan Science Society.

\section{References}

[1] Altes, H. K., Wodarz, D., Jansen V. A. A., 2002. The dual role of CD4 T helper cells in the infection dynamics of HIV and their importance for vaccination, $J$. Theor. Biol., 214, 633-646.

[2] Altes, H. K., Ribeiro, R. M., de Boer, R. J., 2003. The race between initial Thelper expansion and virus growth upon HIV infection influences polyclonality of the response and viral set-point, Proc. Roy. Soc. Lond. B, 270, 1349-1358.

[3] Barber, D. L., Wherry, E. J., Masopust, D., Zhu, B., Allison, J. P., Sharpe, A. H., Freeman, G. J., Ahmed, R., 2006. Restoring function in exhausted CD8 T cells during chronic viral infection, Nature, 439, 682-687.

[4] Binley, J. M., Clas, B., Gettie, A., Vesanen, M., Montefiori, D. C., Sawyer, L., Booth, J., Lewis, M., Marx, P. A., Bonhoeffer, S., Moore, J. P., 2000. Passive infusion of immune serum into simian immunodeficiency virus-infected rhesus macaques undergoing a rapid disease course has minimal effect on plasma viremia, Virology, 270, 237-249.

[5] Ciupe, M. S., Bivort, B. L., Bortz, D. M., Nelson, P. W., 2006. Estimating kinetic parameters from HIV primary infection data through the eyes of three different mathematical models, Math. Biosc., 200, 1-27.

[6] Cecilia, D., Kleeberger, C., Munoz, A., Giorgi, J. V., Zolla-Pazner, S., 1999. A longitudinal study of neutralizing antibodies and disease progression in HIV-1infected subjects, J. Infect. Dis., 179, 1365-1374.

[7] Connolly, N. C., Whiteside, T. L., Wilson, C., Kondragunta, V., Rinaldo, C. R., Riddler, S. A., 2008. Therapeutic immunization with human immunodeficiency virus type 1 (HIV-1) peptide-loaded dendritic cells is safe and induces immunogenicity in HIV-1-infected individuals, Clin. Vaccine. Immunol., 15, 284-292.

[8] Davenport, M. P., Ribeiro, R. M., Perelson, A. S., 2004. Kinetics of virusspecific CD8+ T cells and the control of human immunodeficiency virus infection, J. Virol., 78, 10096-100103. 
[9] De Boer, R. J., Boerlijst, M. C., 1994. Diversity and virulence thresholds in AIDS, Proc. Nat. Acad. Sci. U.S.A., 94, 544-548.

[10] Donaghy, H., Pozniak, A., Gazzard, B., Qazi, N., Gilmour, J., Gotch, F., Patterson, S., 2001. Loss of blood CD11c+ myeloid and CD11c- plasmacytoid dendritic cells in patients with HIV-1 infection correlates with HIV-1 RNA virus load, Blood, 98, 2574-2576.

[11] Donaghy, H., Gazzard, B., Gotch, F., Patterson, S., 2003. Dysfunction and infection of freshly isolated blood myeloid and plasmacytoid dendritic cells in patients infected with HIV-1, Blood, 101, 4505-4511.

[12] Freeman, G. J., Wherry, E. J., Ahmed, R., Sharpe, A. H., 2006. Reinvigorating exhausted HIV-specific T cells via PD-1-PD-1 ligand blockade, J. Exp. Med., 203, 2223-2227.

[13] Galvani, A. P., 2005. The role of mutation accumulation in HIV progression, Proc. Roy. Soc. Lond. B, 272, 1851-1858.

[14] Garcia, F., Lejeune, M., Climent, N., Gil, C., Alcami, J., Morente, V., Alos, L., Ruiz, A., Setoain, J., Fumero, E., Castro, P., Lopez, A., Cruceta, A., Piera, C., Florence, E., Pereira, A., Libois, A., Gonzalez, N., Guila, M., Caballero, M., Lomena, F., Joseph, J., Miro, J. M., Pumarola, T., Plana, M., Gatell, J. M., Gallart, T., 2005. Therapeutic immunization with dendritic cells loaded with heat-inactivated autologous HIV-1 in patients with chronic HIV-1 infection, $J$. Infect. Dis., 191, 1680-1685.

[15] Hogue, I. B., Bajaria, S. H., Fallert, B. A., Qin, S., Reinhart, T. A., Kirschner, D. E., 2008. The dual role of dendritic cells in the immune response to human immunodeficiency virus type 1 infection, J. Gen. Virol., 89, 2228-2239.

[16] Iwami, S., Nakaoka, S., Takeuchi, Y., 2008. Viral diversity limits immune diversity in asymptomatic phase of HIV infection, Theor. Pop. Biol., 73, 332-341.

[17] Iwami, S., Nakaoka, S., Takeuchi,Y., Immune impairment thresholds in HIV infection, In Revision.

[18] Iwasa, Y., Michor, F., Nowak, M. A., 2005. Virus evolution within patients increases pathogenicity, J. Theor. Biol., 232, 17-26.

[19] Janewa, C., Travers, P., Walport, M., Shlomchik, M. J., 2004. Immunobiology: The immune system in health and disease, Garland Pub.. 
[20] Kawamura, T., Gatanaga, H., Borris, D. L., Connors, M., Mitsuya, H., Blauvelt, A., 2003. Decreased stimulation of CD4+ T cell proliferation and IL-2 production by highly enriched populations of HIV-infected dendritic cells, $J$. Immunol., 170, 4260-4266.

[21] Kozyrev, I. L., Ibuki, K., Shimada, T., Kuwata, T., Takemura, T., Hayami, M., Miura, T., 2001. Characterization of less pathogenic infectious molecular clones derived from acute-pathogenic SHIV-89.6P stock virus, Virology, 282, 6-13.

[22] Lore, K., Sonnerborga, A., Brostrom, C., Goh, L.-E., Perrin, L., McDade, H., Stellbrink, H.-J., Gazzard, B., Weber, R., Napolitano, L. A., van Kooyk, Y., Andersson, J., 2002. Accumulation of DC-SIGN+CD40+ dendritic cells with reduced CD80 and CD86 expression in lymphoid tissue during acute HIV-1 infection, AIDS, 16, 683-692.

[23] Mandl, J. N., Regoes, R. R., Garber, D. A., Feinberg, M. B., 2007. Estimating the effectiveness of simian immunodeficiency virus-specific CD8+ T cells from the dynamics of viral immune escape, J. Virol., 81, 11982-11991.

[24] Macatonia, S. E., Lau, R., Patterson, S., Pinching, A. J., Knight, S. C., 1990. Dendritic cell infection, depletion and dysfunction in HIV-infected individuals, Immunology, 71, 38-45.

[25] McKnight, A., Clapham, P. R., 1995. Immune escape and tropism of HIV, Trend. Microbiol., 3, 356-361.

[26] McMichael, A. J., Rowland-Jones, S. L., 2001. Cellular immune responses to HIV, Nature, 410, 980-987.

[27] Meissnera, E. G., Duus, K. M., Gao, F., Yu, X-F., Su, L., 2004. Characterization of a thymus-tropic HIV-1 isolate from a rapid progressor: role of the envelope, Virology, 328, 74-88.

[28] Nowak, M. A., May, R. M., 2000. Virus dynamics, Oxford University Press.

[29] Ogg, G. S., Jin, X., Bonhoeffer, S., Moss, P., Nowak, M. A., Monard, S., Segal, J. P., Cao, Y., Rowland-Jones, S. L., Hurley, A., Markowitz, M., Ho, D. D., McMichael, A. J., Nixon, D. F., 1999. Decay kinetics of human immunodeficiency virus-specific effector cytotoxic T lymphocytes after combination antiretroviral therapy, J. Virol., 73, 797-800. 
[30] Patterson, S., English, N. R., Longhurst, H., Balfe, P., Helbert, M., Pinching, A. J., Knight, S. C., 1998. Analysis of human immunodeficiency virus type 1 (HIV1) variants and levels of infection in dendritic and $T$ cells from symptomatic HIV-1-infected patients, J. Gen. Virol., 79, 247-257.

[31] Perelson, A. S., Neumann, A. U., Markowitz, M., Leonard, J. M., Ho, D. D., 1996. HIV-1 dynamics in vivo: virion clearance rate, infected cell life-span, and viral generation time, Science, 271, 1582-1586.

[32] Regoes, R. R., Wodarz, D., Nowak, M. A., 1998. Virus dynamics: the effect of target cell limitation and immune responses on virus evolution, J. Theor. Biol., 191, 451-462.

[33] Richman, D. D., 2001. HIV chemotherapy, Nature, 410, 995-1001.

[34] Rinaldo, C. R., 2008. Dendritic cell-based human immunodeficiency virus vaccine, J. Int. Med., 265, 138-158.

[35] Smed-Sorensen, A., Lore, K., Walther-Jallow, L., Anderson, J., Spetz, A., 2004. HIV-1 infected dendritic cells up-regulate cell surface markers but fail to produce IL-12 p70 in response to CD40 ligand stimulation, Blood, 104, 2810-2817.

[36] Soumelis, V., Scott, I., Gheyas, F., Bouhour, D., Cozon, G., Cotte, L., Huang, L., Levy, J. A., Liu, Y. J., 2001. Depletion of circulating natural type 1 interferon-producing cells in HIV-infected AIDS patients, Blood, 98, 906-912.

[37] Stafford, M. A., Corey, L., Cao, Y., Daar, E. S., Ho, D. D., Perelson, A. S., 2000. Modeling plasma virus concentration during primary HIV infection, $J$. Theor. Biol., 203, 285-301.

[38] Thieme, H. R., 2003. Mathematics in population biology, Princeton University Press.

[39] Velu, V., Titanji, K., Zhu, B., Husain, S., Pladevega, A., Lai, L., Vanderford, T. H., Chennareddi, L., Silvestri, G., Freeman, G. J., Ahmed, R., Amara, R. R., 2009. Enhancing SIV-specific immunity in vivo by PD-1 blockade, Nature, 458, 206-210.

[40] Wu, L., Kewal-Ramani, V. N., 2006. Dendritic-cell interactions with HIV: infection and viral dissemination, Nat. Rev. Immunol., 6, 859-868.

[41] Wodarz, D., Klenerman, P., Nowak, M. A., 1998. Dynamics of cytotoxic Tlymphocyte exhaustion, Proc. Roy. Soc. Lond. B, 265, 191-203. 
[42] Wodarz, D., Nowak, M. A., 1999. Specific therapy regimes could engender longterm immunological control of HIV, Proc. Nat. Acad. Sci. U.S.A., 96, 1446414469.

[43] Tunetsugu-Yokota, Y., 2005. How does HIV infection destroy the host immune system?, J. AIDS Resear., 7, 171-179. 